\title{
Parametric-Based Path Generation for Automated Vehicles at Roundabouts
}

\author{
David González ${ }^{\mathrm{a}}$, Joshué Pérez ${ }^{\mathrm{b}}$, Vicente Milanés ${ }^{\mathrm{c}}$ \\ ${ }^{a}$ Author is with the Robotics and Intelligent Transportation Systems (RITS) Team, Inria of Paris, 2 rue Simone Iff, 75012 Paris, France \\ email: david.gonzalez@inria.fr \\ ${ }^{b}$ Author is on the Automotive Area / Industry and Transport Division, Tecnalia Research and Innovation, Parque Científico y Tecnológico de \\ Bizkaia, Derio 700, 48160 Derio, Bizkaia, Spain \\ email: joshue.perez@tecnalia.com \\ ${ }^{c}$ Author is with the Research Department, Renault SAS, 1 Avenue du Golf, 78280 Guyancourt, France \\ email: vicente.milanes@renault.com
}

\begin{abstract}
Urban environments are becoming more and more complex because several factors as consecutive crossroads or lanes changes. These scenarios demand specific infrastructures-i.e. roundabouts, for improving traffic flow compared with traditional intersections. A roundabout removes timeouts associated with traffic lights at crossroads and trajectory conflicts among drivers. However, it is a challenging scenario for both humans and automated vehicles. This work presents a path planning method for automated vehicle driving at roundabouts. The proposed system achieves a $G^{1}$ continuous path, minimizing curvature steps to increase smoothness, dividing the driving process in three stages: entrance maneuver, driving within the roundabout and exit maneuver. Parametric equations are generated to deal with automated roundabout driving. This approach allows a real time planning considering two-lane roundabouts, taking different exits. Tests in simulated environments and on our prototype platform-Cybercar-validate the system on real urban environments, showing the proper behavior of the system.
\end{abstract}

Keywords: Roundabouts, automated vehicles, path planning, intelligent transportation systems.

\section{Introduction}

More advanced automated capabilities are being deployed on modern vehicles. Embedded sensor-based driving assistance systems are now able to deal with several traffic situations faster and safer than human drivers do (Van Schijndel-de Nooij et al., 2011). Despite of these remarkable progresses, many efforts have still to be carried out by governments and manufactures for developing systems able to intelligently deal with all the different driving scenarios. Among all these scenarios, roundabouts remain as an unsolved challenge for the development of advanced safety systems. They replace traditional intersections removing the delays associated to traffic lights (Johnson and Hange, 2003), improving the traffic flow. The roundabout is a complex driving scenario, especially for semi-automated and automated vehicles in the oncoming years (Isebrands, 2009).

Roundabouts are relatively recent on urban environments, making difficult for some drivers-e.g. elderly peopleto know how to deal with them ${ }^{1}$. Figure 1(a) shows the proper circulation in function of the exit according to the French road circulation code at roundabouts ${ }^{2,3}$. DARPA Challenges in the United States from 2004 to 2007 are among the most important automated vehicle competitions. Although roundabouts were out of the defined scenarios, several driving functions were shown, including traversing intersections (Ferguson et al., 2009).

First demonstrations including driving through roundabouts were carried out in 2013. Specifically, the PROUD project demo from University of Parma (Italy) on 2013 (Broggi et al., 2013); Mercedes demonstration in Germany covering the so-called Bertha route (Ziegler et al., 2014); and the AUTOPIA urban and peri-urban automated driving demo (Spain) (Godoy et al., 2015). All these demonstrations show several capabilities for automated vehicles in

\footnotetext{
${ }^{1}$ http://www.roundaboutsofbritain.com/

${ }^{2}$ http://www.securite-routiere.gouv.fr/

${ }^{3}$ http://www.mt.public.lu/transports/circulation/code/ 

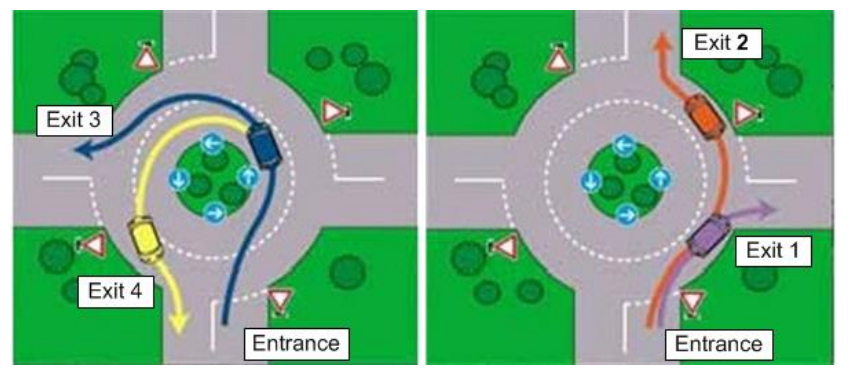

(a) Roundabout behavior taking different exits.

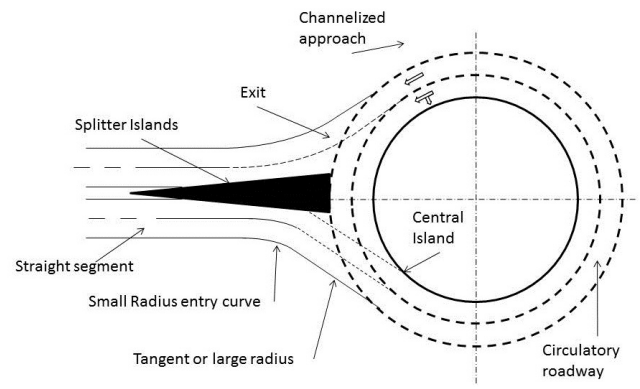

(b) Definitions based on roundabout design in (Rice, 2010b).

Figure 1: Roundabout behavior and definition.

complex scenarios. However, although roundabouts were considered on these routes, they were modeled as standard intersections, without dedicated analysis in this scenario.

In the last two decades, roundabouts have been increasingly considered as a good alternative to address intersections, especially in urban areas (Manage et al., 2003). They provide a number of safety, operational, and other benefits when compared to other types of intersections (Tracza and Chodura, 2012). A detailed definition of the benefits of roundabouts in urban environment-i.e. compact size, operational efficiency, traffic safety and calming, access management, aesthetics and environmental benefits, are presented in (Rodegerdts et al., 2010) and (Rice, 2010a).

From the safety point of view, roundabouts have demonstrated positive effects worldwide. In United States, Robinson et al., (Robinson et al., 2000) concluded that they reduce the crash problems at intersections, approximately by $30 \%$, compared with signalized intersections. In Europe, De Brabander and Vereeck (De Brabander and Vereeck, 2007) showed some conclusions on a Flanders' traffic study between 1994 and 2000, indicating that roundabouts can reduce by $39 \%$ the number of accidents. On the negative side, the driving rules at roundabouts are unknown for some drivers, causing an increment of $28 \%$ of vulnerable road user injuries compared with classical signalized junctions (De Brabander and Vereeck, 2007). This fact turns roundabouts into suitable scenarios for automated driving, maintaining traffic flow benefits and removing safety concerns.

From the traffic flow point of view, there are several studies in the United States and China that show how some drivers do not have enough skills experiencing in roundabouts, generating traffic jams in the vicinities (Abaza and Hussein, 2009; Bai et al., 2009). Roundabouts with and without traffic-signal controls have been intensively studied and widely used (Xiaoguang et al., 2004). Despite of these efforts, roundabout applications have been only considered from the management point of view, providing reference speed control (Bai et al., 2009; Molinete et al., 2009; Yu and Qin, 2009).

From a classification point of view, a roundabout-dedicated Federal Highway Administration (FHWA) Technical Summary (Rodegerdts et al., 2010) divided them according to the size and number of lanes having: 1) Miniroundabouts-a type of intersection that can be used at physically-constrained locations in place of stop-controlled or signalized intersections to help improve safety problems and reduce excessive delays at minor approaches (Robinson et al., 2000); 2) Single-lane; and 3) Multi-lane roundabout. This work is focused on the second and third types but results can be easily adopted in mini-roundabouts without any extra complexity.

Path and trajectory planning research has been very active on the intelligent vehicles field (see (González et al., 2016; Paden et al., 2016) for state-of-the-art review) in last years. However, trajectory generation in roundabouts has not been further investigated, specially in real environments. Two main demonstrations have effectively tackled roundabouts: 1) Broggi et al. (Broggi et al., 2014) presented the PROUD test, involving different driving scenarios as highways, rural roads and urban roads (involving 6 roundabouts). The planning stage was composed of a database of precomputed clothoids, from which the best path was selected, targeting the center of the lane. The road layout was detected implementing vision algorithms, as well as obstacles and other road users; and 2) Ziegler et al. (Ziegler et al., 2014) developed a planner that was based on global waypoints passed through a numerical optimization approach to plan its way through urban environments and perform the Bertha-Benz memorial route, managing several roundabouts. 
Stereo cameras detected the road layout, the obstacles and other road users.

This paper presents a general approach using geometrical roundabout definitions in a digital map (center in 2dimensional Cartesian coordinates, the radius, the number of lanes and the entrances and exits) to build a real-time reference path using parametric equations. The path is then given to the control stage where a lateral controller is able to follow the geometrical reference. Although, different control architectures for automated vehicles have been proposed in the literature, most of them consider perception, decision and control stages as the key ones (González et al., 2016). Our approach can be easily integrated in the decision and path planning modules, because its modularity permits an adaptability of the algorithm to any automated vehicle architecture. The system was firstly tested on simulation; then, tests on real roads were performed, providing a real-time trajectory planning generation for roundabouts.

This work is based on previous research (see (González and Pérez, 2013) and (Perez Rastelli et al., 2014) for details), the main contributions are:

- G1 continuous roundabout entrance and exit by using an improved generation of the parametric control points.

- Path continuity, curvature smoothness and comfort constraints considerations for in-roundabout driving.

- Real time path generation considering two-lane roundabouts, including lane-change and taking different exits.

- The implementation of the algorithm in a real roundabout using a real electric platform.

The remainder of the paper is structured as follows. Section II presents a review of the definitions and constraints for the roundabouts, and gives an overview of the approach adopted, based on parametric curves generation. The automated roundabout algorithm will be described in section III, based on the path generation and the curve generation process. The simulator, the prototype vehicle and the test environment used for the validation of this proposal will be highlighted in section IV. Some of the results will be compared with simulations and real driving tests and discussed in section V. Finally, section VI presents some concluding remarks and future works.

\section{Roundabouts: definitions and constraints}

This section provides a comprehensive review about possible configurations on roundabouts, according to the size, the number of lanes and geometrical characteristics. Then, a detailed description about the considerations to take into account when it comes to autonomously drive through a roundabout is given.

\subsection{Definitions}

A roundabout can be described as a composition and convergence of several intersections. According to the specific driving area, some parameters may vary as: number of entrances/exits, lanes, speed limit, geometric design or path alignment. A detailed review about the roundabout types is found in (Rice, 2010b). Our proposal faces the roundabout driving for the planning and control point of view, identifying three stages entrance, circulatory roadway and exit (see Fig. 1(b)) as follows:

- Entrance: where the entry curve is generated. It covers the route from the straight stretch up to the beginning of the circular area. It can be divided in two sub-stages: the small radius entry and the tangent or large radius (see Figure 1(b)).

- Circulatory roadway: is defined as the middle stage, where generally circular shapes are used (Rice, 2010b). It can be composed by one or multiple lanes, forcing lane-change maneuvers when entering/exiting the inner lane.

- Exit: is the final stage and it allows the vehicle to leave the roundabout. This stage is complementary to the entrance, having the same sub-stages: first the tangent or large radius and then the small radius exit.

Other parameters can significantly affect the automated roundabout path generation. Some of them are: 1) The central island, i.e. the raised zone defined in the center of the roundabout. It defines the minimum turning radius in the circulatory roadway; 2) Splitter islands, used in the entrance and exit of roundabouts, forcing the reduction of the entry speed; and 3) Channelized approach, describing dedicated exits in the roundabout to improve traffic flow. Other traffic rules (e.g. U.K.), are easily tunable due to the modular characteristics of parametric curves used in the approach (i.e. circle and Bézier equations). 


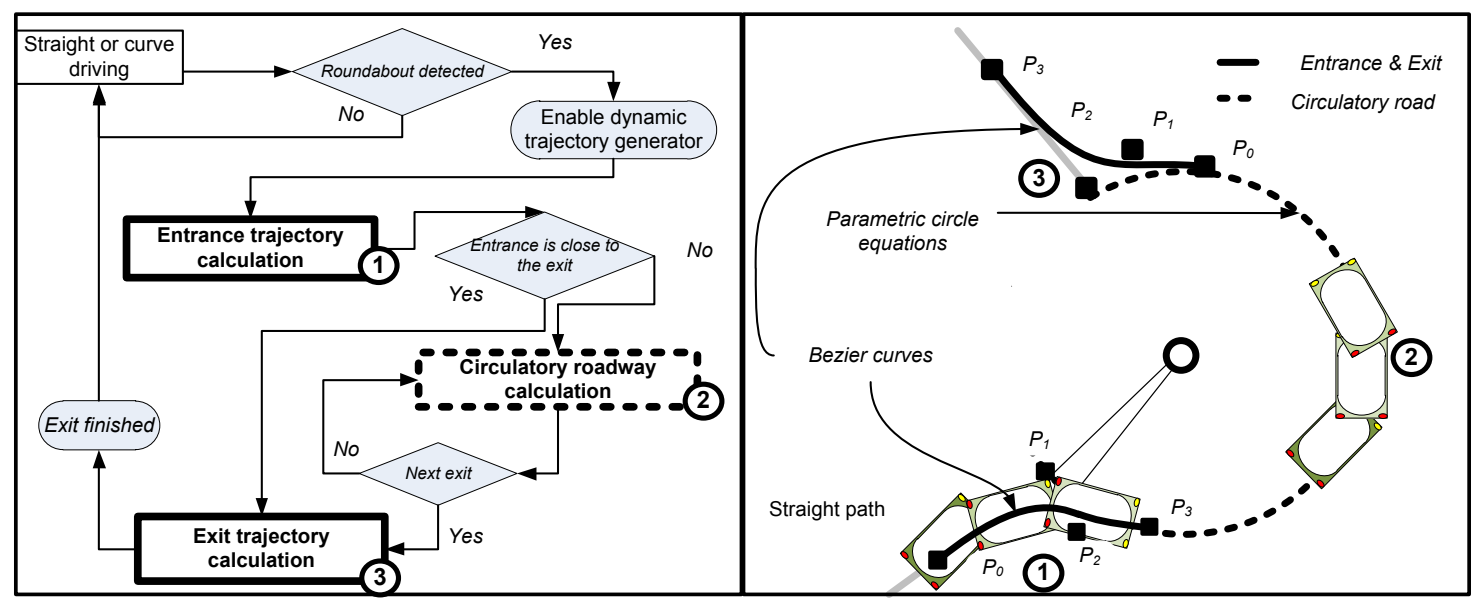

Figure 2: Roundabout phases and parametric path generation.

\subsection{Constraints}

There are some constraints to the proposed method imposed by both, the physical limitations of the experimental platform and the infrastructure design. Below, the main characteristics of type of roundabouts considered in this work are:

- The maximum turning radius in the roundabout - considering the inner lane- has to be equal or bigger to the minimum turning radius of the vehicle with 3 meter width per lane. For the experimental vehicle used in this work, the minimum internal turning radius is 7.0 meters.

- The circulatory roadway is formed by two lanes and the roundabout comprises four exits.

- Perpendicular and tangential roads to the large radius of the roundabout are used.

- The interaction with additional vehicles - either automated or not-is out of the scope of this work. The reader is referred to (Milanés et al., 2012) and (Muffert et al., 2012) for possible approaches addressing intersection interaction.

- The reference speed is defined in function of the current stretch in which the vehicle is. It is defined based on several parameters as the curvature and comfort level in the driving process (see (Perez Rastelli et al., 2014) for details).

Based on Sections 2.1 and 2.2, the following details the design and development of a path planning algorithm for automated vehicles in roundabouts. This approach is included into the Inria control architecture for automated vehicles (González and Pérez, 2013). This algorithm is able to create a real time path for dealing with any complex scenario as roundabouts. Simulations and real experiments prove the efficiency of this proposal.

\section{Automated Roundabout Algorithm}

The proposed algorithm is developed within a global architecture for automated vehicles previously developed (see (González and Pérez, 2013) for details) where modularity plays a key role. The main contributions of this work address decision and control stages. Moreover, the information coming from the perception stage is used to know in real time the vehicle location and the obstacles around. The decision stage uses this information, based on the defined global planner, to generate a local path. Finally, the control stage follows the real-time generated path, being able to autonomously drive through different intersections, specifically roundabouts. 
In (Perez Rastelli et al., 2014), a description of the curve generation process used for intersections is presented. This process divides the interpolating curve to provide continuous paths for the vehicle to follow. The algorithm uses reference points, e.g. the middle points of crossroads and the distance to the sidewalks, the road width and vehicle kinematic to compute the different stretches. A geometrical approach that considers the joint points between straight and curve segments was proposed. This algorithm cannot be extrapolated to roundabouts because it presents singular constraints (see Sec. II for details). The main contribution of this work is an automated path planning algorithm, based on parametric equations, for smooth path generation and tracking in roundabouts. Our approach considers several parameters as the vehicle dimensions, speed limit, comfort accelerations and characteristic of the infrastructure (i.e. small radius entry curve, tangent radius and central island). Joints between different segments in the path are at least $G^{1}$ (geometrically continuous). Next sections describe the implementation carried out in the decision stage, based on the path generation process in roundabouts and the control laws used for the tracking.

\section{1. $G^{1}$-continuous path generation}

To meet roundabouts special shape, the path planning was divided in three stages: entrance, circulatory roadway and exit (see Figure 2). The left part of this figure shows the flowchart of the proposed algorithm. The first and the last stages can be modeled as standard intersections, but considering the small radius entry curve and the tangent radius, based on parametric equations as in (González and Pérez, 2013). The junction points require special attention to avoid discontinuities on the path. The circulatory roadway can be modeled as in (Pérez et al., 2011), using circular parametric equations, but also considering lane changes.

The definition of entrance and exit segments is based on Bézier curve generation. The polynomial is described as follow:

$$
\mathbf{B}(t)=\sum_{i=0}^{n} \mathbf{P}_{i} \mathbf{b}_{i, n}(t) ; \quad t \in[0,1]
$$

where $n$ is the degree of the polynomial, and $\mathbf{P}_{i}$ are the control points (see Figure 2), and $\mathbf{b}_{i, n}$ is the Bernstein basis polynomial given by:

$$
\mathbf{b}_{i, n}(t)=\left(\begin{array}{c}
n \\
i
\end{array}\right)(1-t)^{n-i} t^{i}
$$

In this work, Bézier curves of third and fourth degree are implemented according to geometrical characteristics for the entrance and the exit of the roundabout.

$$
k(t)=\frac{\mathbf{B}^{\prime}(t) \times \mathbf{B}^{\prime \prime}(t)}{\left\|\mathbf{B}^{\prime}(t)\right\|^{3}}
$$

Equation 3 presents the computation of the curvature for $B_{(t)}$, being $B^{\prime}(t)$ and $B^{\prime \prime}(t)$ the first and second derivative of the Bézier curve $B_{(t)}$.In Bézier curves $t$ represents the parameter defined between [0,1]; an iterative process generates the curve at each entry or exit stage. Right part of Figure 2 shows a vehicle in the three stages proposed for the roundabout. Once the entrance of the roundabout is detected, the first parametric equation is generated up to the tangent line of the circle. In this stage, two conditions are evaluated: if the exit is close, then the second stage is omitted, jumping directly to final stage. The circulatory roadway is generated using the circle parametric generation, as in (Pérez et al., 2011).

Many techniques, as splines (Labakhua et al., 2008), clothoids (Brezak and Petrovic, 2014) and polynomials (Piazzi et al., 2002), can be used for the path planning in this scenario. However, Bézier shows several advantages, as presented in previous works (Hand et al., 2010; González and Pérez, 2013). These are resumed as follow:

- The control points $P_{0}$ and $P_{n}$ define the beginning and the end of the curve, respectively.

- The tangent vector formed by $\overrightarrow{P_{0} P_{1}}$ defines the initial direction of the curve, as well as $\overrightarrow{P_{n-1} P_{n}}$ defines the ending direction at the end of the curve.

- The control points define the convex hull area (the Bézier curve lies within). 


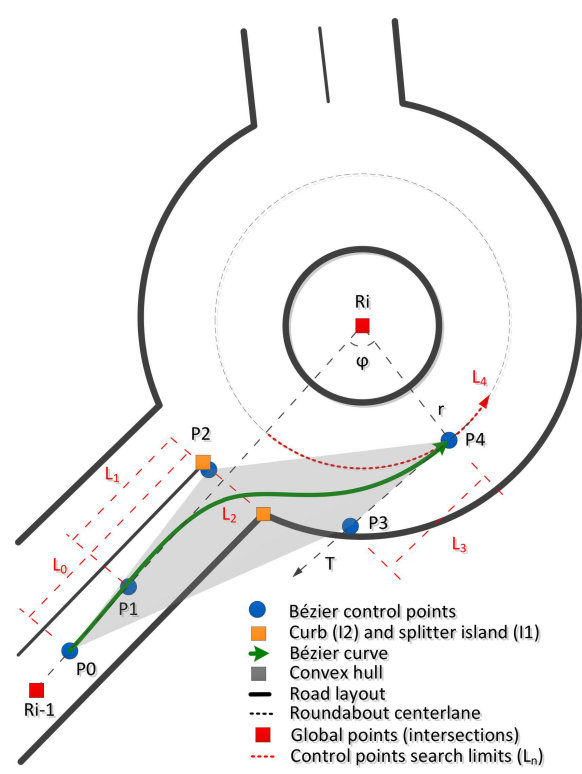

(a) Solution for the roundabouts.

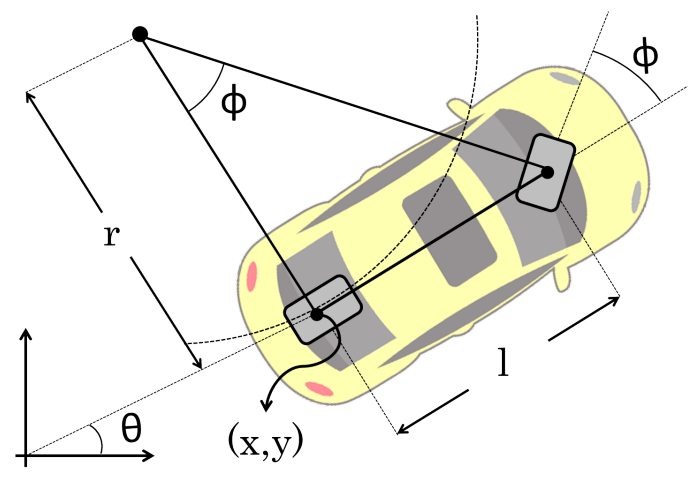

(b) Kinematic model of Ackerman.

Figure 3: Roundabout entry solution and vehicle model.

Depending on the roundabout, different Bézier degree polynomials are generated. First, $3^{r d}$ degree Béziers are tested according to an optimization function that takes into account the curvature of the generated Bezier curve, and the curvature error in the joining points of two curves. If no curve meets the requirements (not complying with $\mathrm{road} /$ vehicle constraints), $4^{\text {th }}$ degree Béziers are implemented in a similar process to find the best curve. The extra control point coming from the curve degree elevation (P2 point in Figure 3(a)) permits to pull the curve closer to the splitter island, introducing more flexibility in the path and enabling configurations unreachable with $3^{\text {rd }}$ degree Béziers. Figure 3(a) shows an example of the path generation with a $4^{\text {th }}$ degree Bézier, where point $P 2$ is moved in-between the line generated from the curb to the splitter island (yellow squares), thus forcing the Bézier to stay within bounds (see the convex-hull in grey), while still pulling the curve towards the splitter island.

The proposed algorithm sets the position of Bézier control points in a dynamic way, assuring a safety path generation. It is a resolution complete planner, meaning that the optimality of the solution will depend on the discretized search of the control points position (if a solution exists in the current control points search resolution, the planner is able to find it). In case the curve does not exist, the planner notifies control and decision stages (requesting safety measurements and a new strategy, respectively).

Algorithm 1 describes the path generation, where $k$ is the curvature of the evaluated point in the curve at a given iteration (i.e. Equation 3 at a given $t$ ) and $k_{\max }$ is the maximum curvature value. $k_{\text {start }}$ and $k_{\text {end }}$ are the curvature continuity errors at $P_{0}$ and $P_{n}$ respectively, these are computed to assess the continuity of the curve w.r.t. adjacent stretches, e.g. straight segments before the roundabout and curve segments inside the roundabout (see Fig. 3(a)). The parameter $n$ is the Bézier degree. These data are stored in an dynamic array, allocating a reward if the value of $k_{\max }$ is lower than the vehicle's maximum feasible $k$. The best option is thus the one with the smallest reward value (minimizing the continuity error between segments).

\subsection{Curve generation process in roundabouts}

Figure 3(a) shows the characteristics of the path generation at roundabouts. In the entrance of the roundabout, the small radius entry curve, the tangent radius and the radius of the circulatory roadway are considered in the path generation.

In this sense, the splitter island's position limits the Bézier convex-hull (gray region in Figure 3(a)) which gives a secure internal band to the vehicle since the curve lies within the hull (Perez Rastelli et al., 2014). Points $P_{3}$ and 


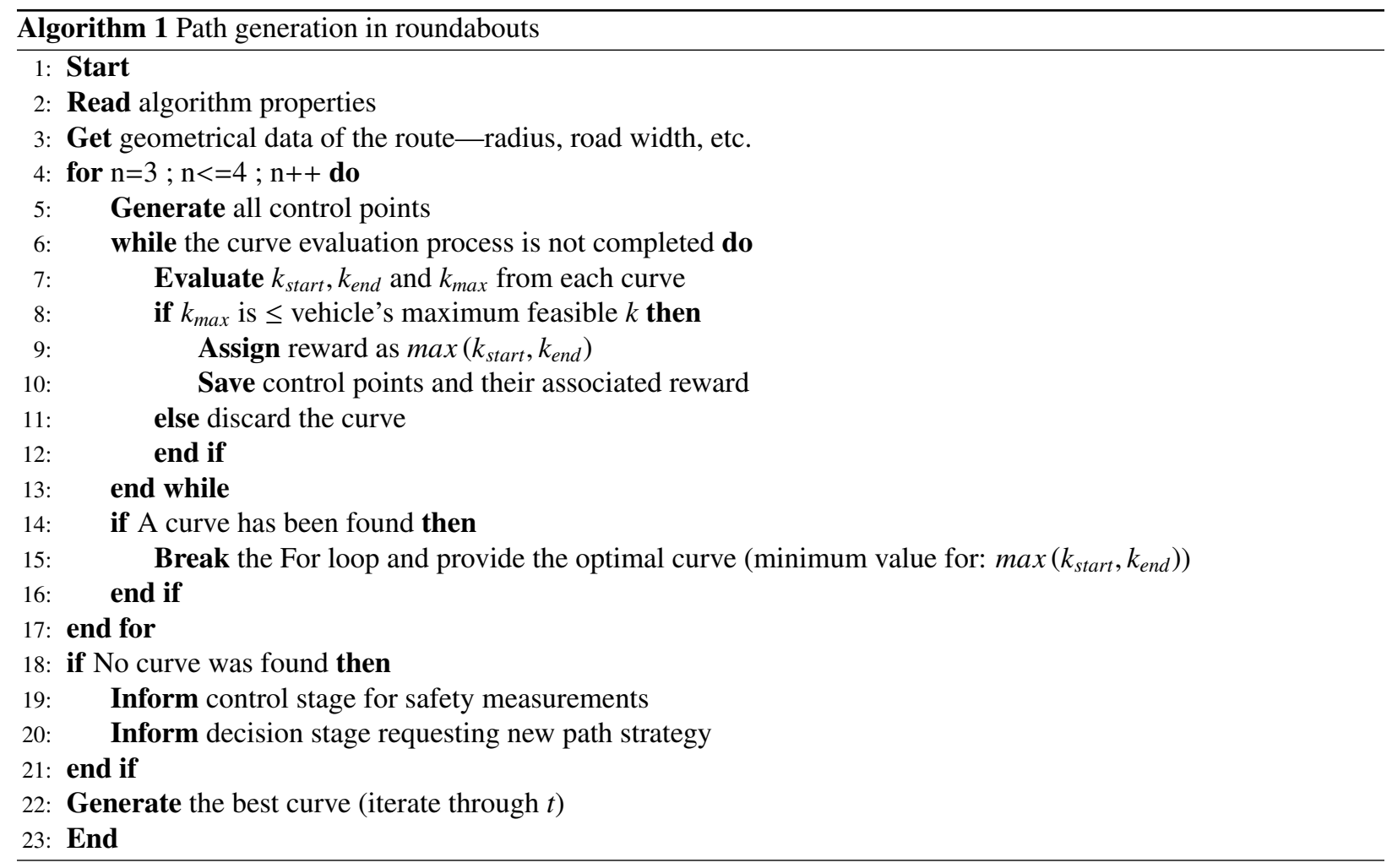

$P_{4}$ are set over the tangent line to the circulatory roadway to ensure soft curvature transitions in the path. The vector defined by $\overrightarrow{P_{0} P_{1}}$ describes the direction of the entry road in Figure 3(a), being $\overrightarrow{P_{3} P_{4}}$ the points defining the exit stretch when leaving the roundabout. The generation of the control points is as follow:

- The yellow points in Figure 3(a) describe the road layout ( $I_{1}$ for the splitter island and $I_{2}$ for the curb), constraining the movement of point $P_{2}$ and ensuring the convex-hull does not surpasses the splitter island (the point lies within the line formed by these yellow points).

- The distance from the curve (green) to the curb and the splitter island (yellow squares) has to be at least half of the vehicle's with. If not, the curve is discarded (avoiding going over the curb).

- The maximum curvature in the Bézier curve must be less than the maximum physical curvature of the vehicle, defined in Equation 3.

Figure 3(a) shows the area where the control points (from $P_{0}$ to $P_{4}$ ) are established. The position of the point $P_{0}$ is calculated as in Equation 4 and $P_{4}$ is represented by the Equation 8:

$$
\begin{gathered}
P_{0}=R_{i}+L_{0} \frac{R_{i-1}-R_{i}}{\left\|R_{i-1}-R_{i}\right\|} \\
P_{1}=R_{i}+L_{1} \frac{R_{i-1}-R_{i}}{\left\|R_{i-1}-R_{i}\right\|} \\
P_{2}=I_{1}+L_{2} \frac{I_{1}-I_{2}}{\left\|I_{1}-I_{2}\right\|} \\
P_{3}=P_{4}+L_{3} \vec{T}
\end{gathered}
$$




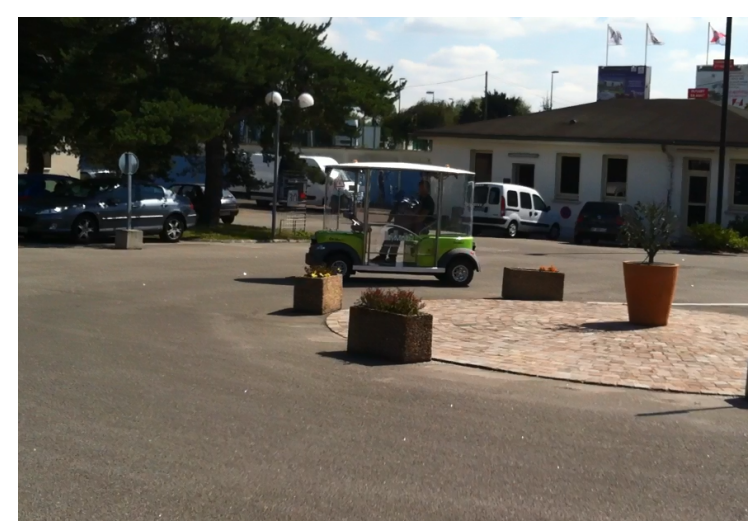

(a) Roundabout and Cyberbus on real implementation.

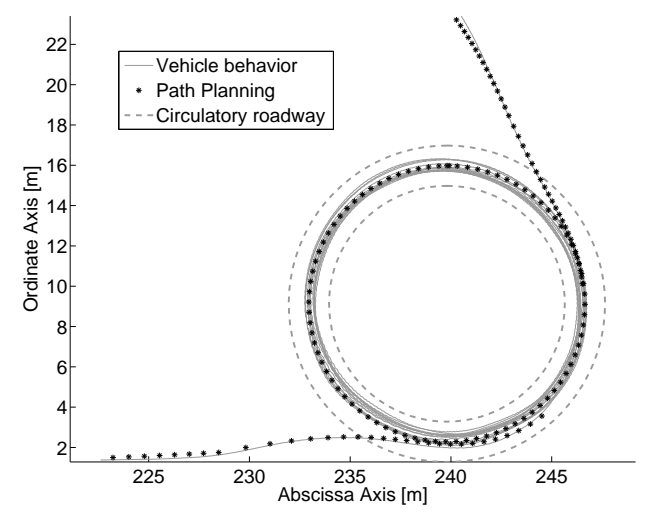

(b) Adjusting the controller inside the roundabout.

Figure 4: Real platform and controller adjustments.

$$
P_{4_{(x, y)}}=\left(\begin{array}{l}
r \cos \left(\arctan \left(\frac{R_{i-1 y}-R_{i y}}{R_{1-1 x}-R_{i x}}\right)+\varphi\right)+R_{i x} \\
r \sin \left(\arctan \left(\frac{R_{i-1 y}-R_{i y}}{R_{i-1 x}-R_{i x}}\right)+\varphi\right)+R_{i y}
\end{array}\right)
$$

where $L_{0}$ and $L_{1}$ is the linear separation between $P_{0}, P_{1}$ respectively from $R_{i} . R_{i}$ and $R_{i-1}$ define the roundabout center and the previous intersection respectively. Please notice that $L_{0}$ is always greater than $L_{1}$ to avoid unexpected curve behaviors and both, $L_{0}$ and $L_{1}$, are always greater than the external radius of the roundabout. $L_{2}$ describes the position of $P_{2}$ inside the line $\overrightarrow{I_{1} I_{2}}$. Moreover, $P_{3}$ is defined by $L_{3}$ which is the distance from $P_{4}$ to $P_{3}$ in the direction of vector $\vec{T}$, which is a vector tangent to the roundabout at $P_{4}$. Please also notice that if only $3^{r d}$ degree curves are needed, then $P_{2}$ is removed. Moreover, $\varphi$ is defined by Equation 9:

$$
\varphi=\frac{L_{4}}{r}
$$

where $L_{4}$ is the segment of arch formed between $\vec{R}_{i-1} R_{i}$ and $P_{4}$, and $r$ is the radius of the roundabout (Circulatory roadway). The exit of the roundabout is managed as an entrance, using symmetrical conditions. The circulatory roadway segment of the roundabout is generated by parametric circle equations, as in (Pérez et al., 2011). Having this in mind, line 5 in Algorithm 1 generates all the curves to be evaluated. The control points are constrained to linear search spaces (defined by $L_{0}, L_{1}, L_{2}, L_{3}$ and $L_{4}$ ). The curve generation is done iteratively, being the limits and resolution for each of the search spaces as follows:

- For the external control points $P_{0}$ and $P_{4}, L_{0}$ will be constraint at maximum $20 \mathrm{~m}$ from the external radius and $L_{4}$ at $20 m$.

- For $P_{1}, L_{1}$ will be constraint at maximum $L_{0}$ distance from the external radius (forcing $P_{1}$ to always be further into the intersection than $P_{0}$, avoiding undesired behaviors). For $P_{3}, L_{3}$ will be limited to $L_{n}$.

- If $P_{2}$ exists ( $4^{\text {th }}$ degree Béziers), $L_{2}$ will be constraint to $\left\|\overrightarrow{I_{1} I_{2}}\right\|$.

- Outer control points related to $L_{0}$ and $L_{4}$ will have a resolution of $2 m$ (10 iterations per control point). Inner control points length $L_{1}$ and $L_{3}$ will also be covered in 10 iterations, having variable resolution (since they are limited by $L_{0}$ and $L_{4}$ respectively. Finally, $L_{2}$ is covered in 5 iterations, presenting also a variable resolution depending on $\left\|\overrightarrow{I_{1} I_{2}}\right\|$. 


\subsection{Kinematic Model and lateral control law}

Most of the roundabouts in urban scenarios are limited to $30 \mathrm{~km} / \mathrm{h}$ (Rodegerdts et al., 2010). For this reason, a kinematic model is considered for the path generation algorithm. Vehicle's characteristics have been used for motion model in real implementation, as in (Piazzi et al., 2002) and (Sotelo, 2003). This bicycle model is also considered for the control law.

The differential equations, describing the movement in a Cartesian plane (see Figure 3(b)), are as follow:

$$
\left\{\begin{array}{l}
\dot{x}(t)=\frac{d x(t)}{d t}=V_{t} \cos (\theta(t)) \\
\dot{y}(t)=\frac{d y(t)}{d t}=V_{t} \sin (\theta(t)) \\
\dot{\theta}(t)=\frac{d \theta(t)}{d t}=\frac{V_{t}}{l} \tan (\phi(t))
\end{array}\right.
$$

where $\theta$ is the orientation angle with respect to plane $x, y, \phi$ is the steering angle of the front wheel, $L$ is the wheel base and $V_{t}$ is the longitudinal speed. The point $(x, y)$ are defined with respect to the center of the rear axle of the vehicle. Due to the low speed at roundabouts, the centrifugal force is considered as despicable (no slide angles nor slip angles are considered); the wheel slipping and the forces transferred between wheels of the same axle track are approximated to zero. Thus, we assume that the maximum feasible curvature is when the turning angle of the wheel is maximum. The control law is based on classic control variables for automated vehicles; such as lateral error, angular error and the curvature (Naranjo et al., 2009). It is defined as follows:

$$
U(t)=\alpha_{1} k(t)+\alpha_{2} L_{\text {error }}+\alpha_{3} A_{\text {error }}
$$

where $k(t)$ is the curvature, $L_{\text {error }}$ is the lateral error and $A_{\text {error }}$ is the angular error. And $\alpha_{1}, \alpha_{2}$ and $\alpha_{3}$ are the controller gains. These errors are calculated w.r.t. the look-ahead control point (placed at the frontal bumper of the vehicle) and the reference path. The $L_{\text {error }}$ has a proportional effect for the control action, since it is associated to the reference error in $y$. Moreover, $A_{\text {error }}$ has a derivative influence $\left(\frac{d y}{d t}\right)$ based on the information of Equation 10. According to the control law, it is a Proportional Derivative (PD), where $\alpha_{2}$ reduces the lateral error and $\alpha_{3}$ avoids oscillations when the vehicle is inside the roundabout. This controller allows a faster and smooth lateral action on these kind of scenarios.

Figure 4(b) shows the final tuning of the controller for the real roundabout. Several turns were performed at difference speeds (between 1.0 and $4.0 \mathrm{~m} / \mathrm{s}$ ). The maximum error valued is around $15 \mathrm{~cm}$, which is low, compared to the SLAM error (Xie et al., 2010). Regarding the comfort while driving inside the roundabout, there is not oscillation, and due to the speed range used in the experiment, the lateral acceleration never exceeds $1.0 \mathrm{~m} / \mathrm{s}^{2}$-Fairy uncomfortable-based on the ISO 2631-1 Standard (ISO, 1997).

\section{Simulator, Prototype vehicle and Test environment}

Modularity is the one most important criterion in the design of the proposed control algorithms. The software environment used for the implementation is RTMaps ${ }^{4}$, a real-time tool, designed for fast and robust implementation in multitasks and multisensor data. It interfaces both, simulations and real platforms, based on a multi-thread structure. Only the acquisition and actuators components are adapted, as in (Gonzalez et al., 2014), while all algorithms remains identical.

\subsection{Simulator}

Pro-SiVIC is a software environment for the assessment of the robustness and reliability of on-board sensors. It has a multi-sensorial environment, considering real car parameters such as the inertia, steering wheel response or lateral acceleration with yaw angles and it is compatible with RTMaps. RITS control architecture is implemented on this simulator (Gonzalez et al., 2014). Experiments were first validated using Pro-SiVIC which allows implementing a virtual vehicle in urban scenario, including roundabouts.

\footnotetext{
${ }^{4}$ http://www.intempora.com/
} 


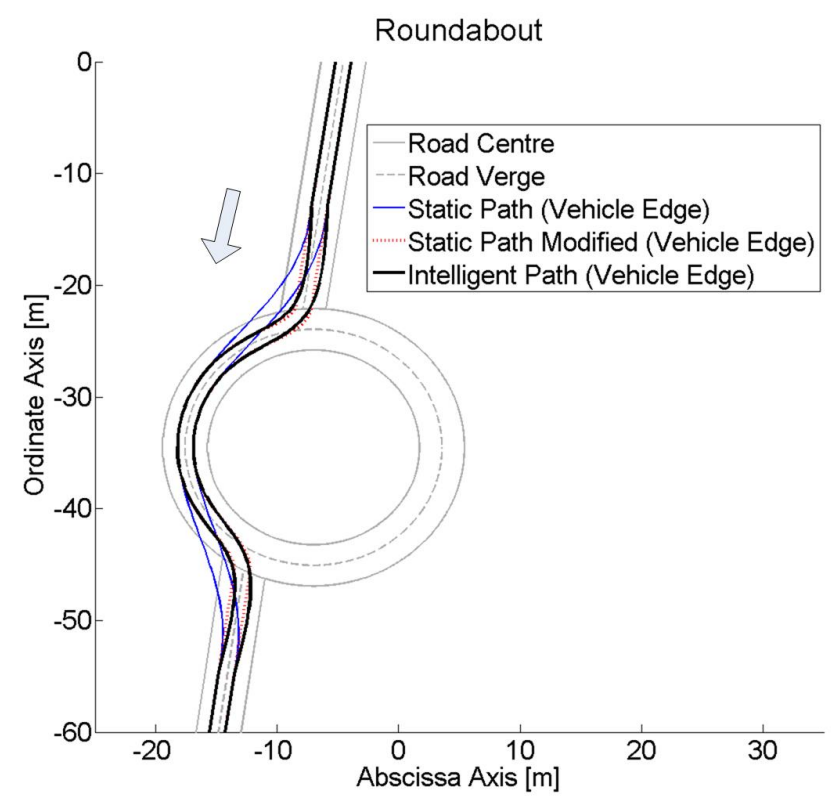

(a) Roundabout

Curvature on Roundabout

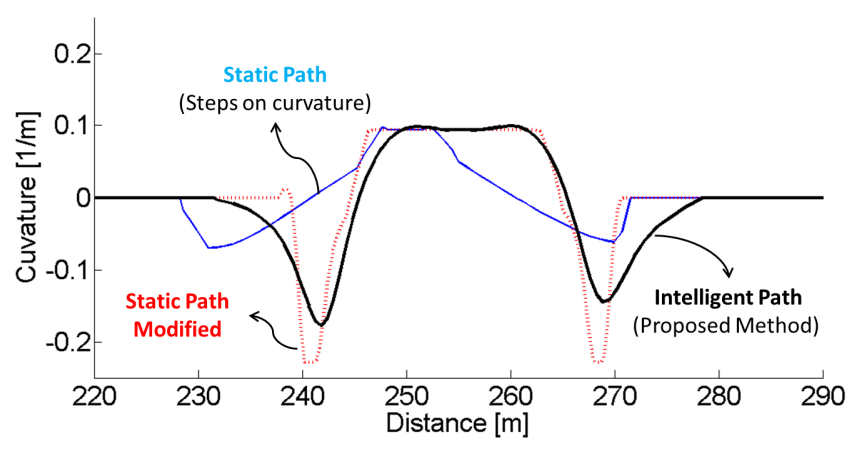

(b) Curvature

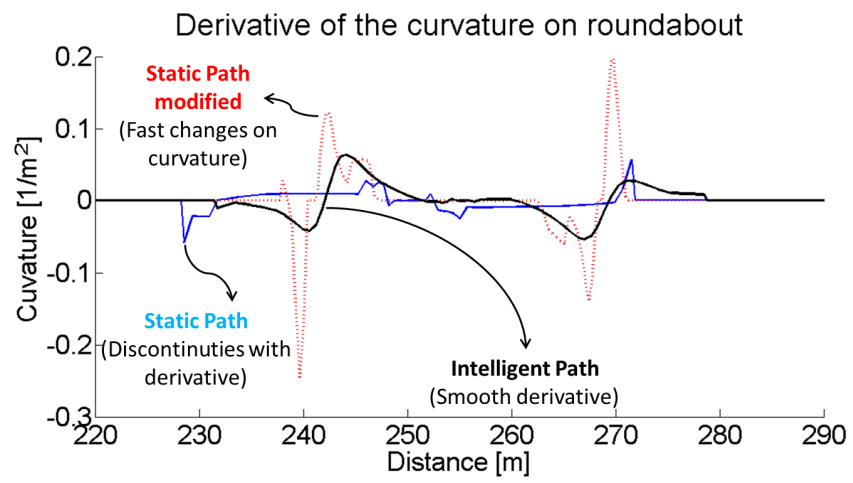

(c) Derivative of the curvature

Figure 5: Behaviour over the Roundabout using different path planning methods 


\subsection{Prototype vehicle}

The prototype used is an electric minibus system without mechanical driving actuators. It is capable of traveling autonomously from one point to another in a road network. It has been used in some European project demonstration, as CityMobil and CityMobil II (Bouraoui et al., 2011; Roldao et al., 2015).

Simultaneous Localization and Mapping (SLAM) fused with an Inertial Measurement Unit (IMU) is used for the localization of the vehicle. The positioning algorithm is capable of estimate the vehicle location using real time information (see (Trehard et al., 2014) for details). Figure 4(a) shows the prototype vehicle.

\subsection{Test environment}

Figure 4(a) shows test track used for the real experiments. The facilities are at INRIA-Rocquencourt, France. The main characteristics of this roundabout are:

- The radius of the roundabout is 8.5 meters.

- It has four entrances/exits, 3 meters wide each.

- The roundabout has two lanes inside, 3 meters each.

- The flow direction is counter-clockwise.

\section{Experiments}

Experiments are focused on showing the path planning generation for the three phases on the roundabout. Different entrances and exits of the roundabout were carried out, including lane change inside the roundabout. The lateral acceleration, the curvature, its derivative and the lateral and angular errors are used for evaluating the proposed method.

\subsection{Simulations}

Simulations were carried out with RTMaps and ProSivic (see section 4). Figure 5 shows a comparison using three different methods: 1) based on static path generation (blue line); 2) based on the introduction of road constraints for path generation (González and Pérez, 2013) (dotted red line); and 3) the algorithm proposed in this paper (black line). The road limits and the road verge are depicted in gray and dotted gray respectively.

The first method was tested on curve stretches giving a proper behavior. It fixes the polynomial control points to a certain distance. One can appreciate how such generation causes that the generated path to cross over the sidewalk. The second method (dotted red line) modifies the control points location according to the road constraints, forcing the path to be into the road. This second approach causes a discontinuity on the curvature (see 5(b)) that is also reflected on its derivative 5(c). The last method automatically sets the control points based on Bézier properties and constraints explained in section 3 , achieving a smoother path planning.

Figures 5(b) and 5(c) show the curvature and their derivative per each experiment. The static path generation (blue line) presents fewer but more abrupt changes. The second algorithm (dotted red line) shows a curvature overshoot (more pronounced in its derivative), causing higher lateral accelerations and discomfort. Finally, the proposed method (black line) shows a better behavior for changes of the curvature, improving the comfort.

Figure 6 shows the behavior of the proposed algorithm when taking different exits using both inner and outer lanes. For the scenario when the first exit is taken, the path only considers Bézier curves as in a standard intersection. This is because the exit is too close to the entrance, making the circulatory roadway negligible. The other two trajectories depict the scenario when the second exit is taken. Despite the external lane is used when taking the second exit, the use of the inner lane allows to show the curvature evolution.

Figure 6(c) shows the curvature in the three scenarios shown in Figure 6(a). For the first case, the curvature is smoother and smaller than in the second and third cases. It is because the behavior is similar than a standard intersection and there are no inflection points in the curvature. However, the curvature is a little higher when taking the inner lane. Experiment 2 shows the curvature generation taking the external lane. The derivative change has a 


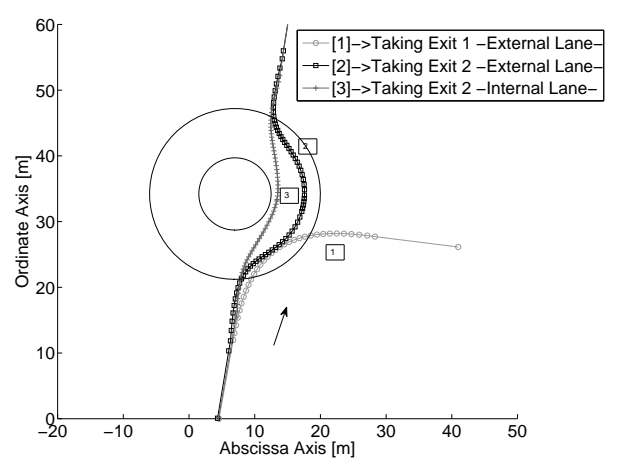

(a) Planning in the Roundabout -Exit 1 and 2-

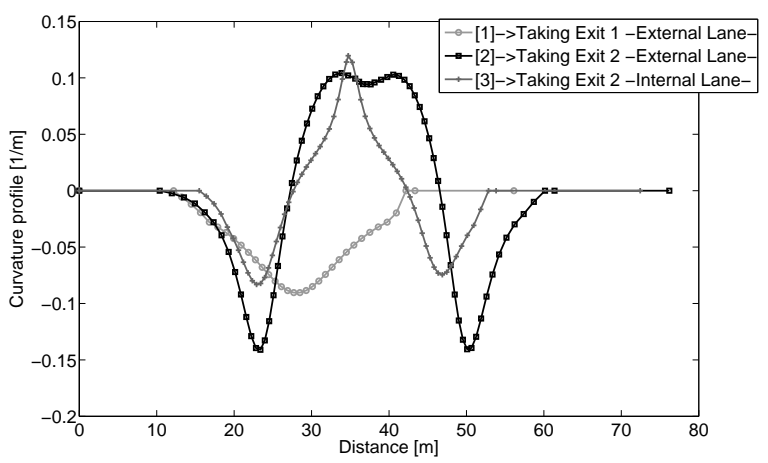

(c) Curvature -Exit 1 and 2-

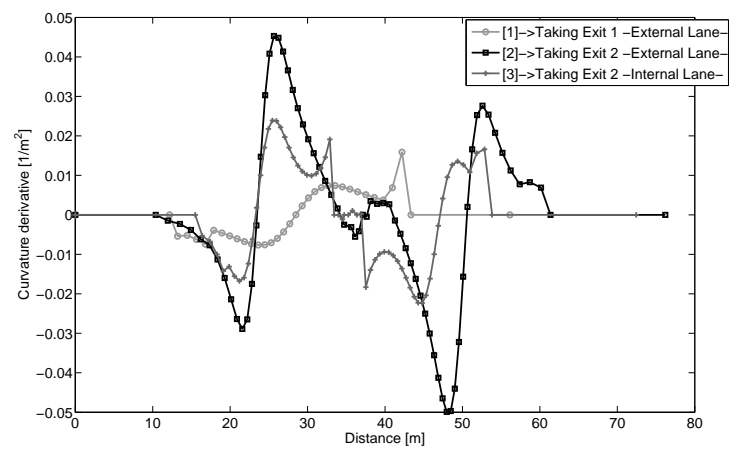

(e) Derivative of the curvature -Exit 1 and 2-

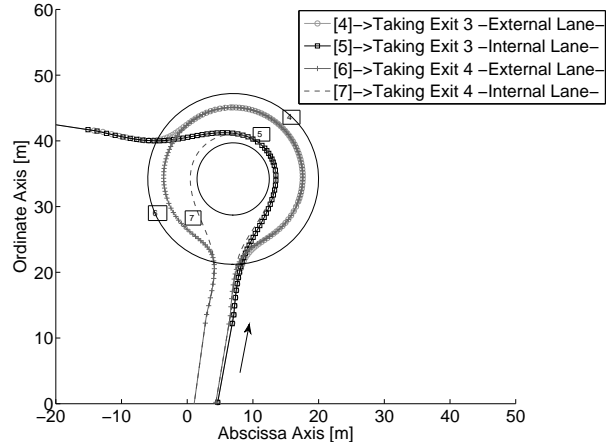

(b) Planning in the Roundabout -Exit 3 and 4-

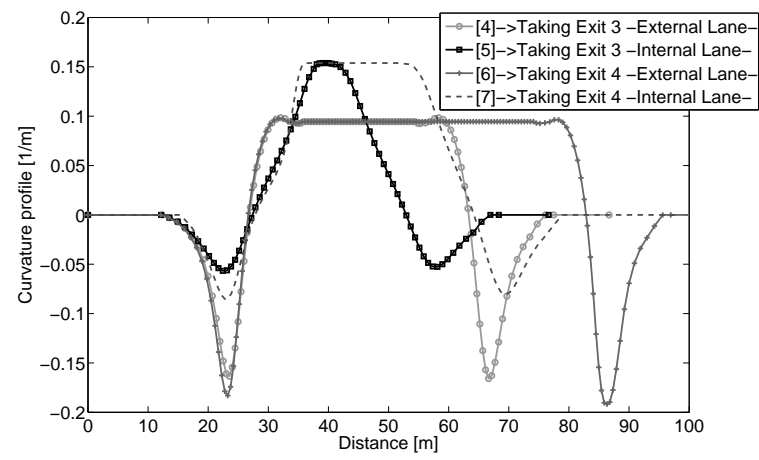

(d) Curvature -Exit 3 and 4-

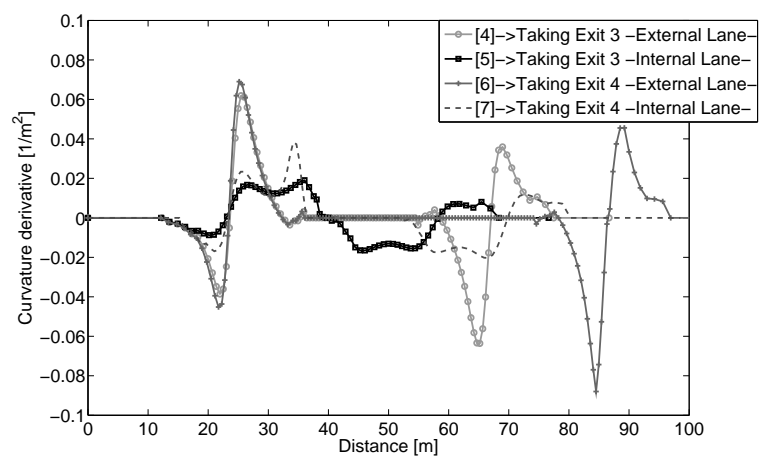

(f) Derivative of the curvature -Exit 3 and 4-

Figure 6: Experiments with different radii, lane changes and exits

better behavior on the experiment 3 (see Fig. 6(e)). This shows how the comfort of the drivers is better in the third case, because the reduction of the lateral accelerations inside the roundabout.

Experiments taking the third and fourth exits following the French Road Circulation Code are shown in Figures 6(b),6(d) and 6(f). Figure 6(b) shows the Cartesian position of the trajectories generated. Curvature and its derivative are shown in figure 6(d) and 6(f) respectively. At the beginning of Figure 6(d), the entrance of the roundabout is shown. When the outer lane is taken (see tests 4 and 6 in Figure 6(b)), the the vehicle steers more than when taking the inner lane (i.e. tests 5 and 7 in Figure 6(b)), causing a higher curvature in the entry and exit stages. In both cases, 
the use of the inner lane has a better behavior. The covered distance is smaller (see Fig. 6(d)) and the derivative of the curvatures have smoother changes during the whole maneuver (see Fig. 6(f)).

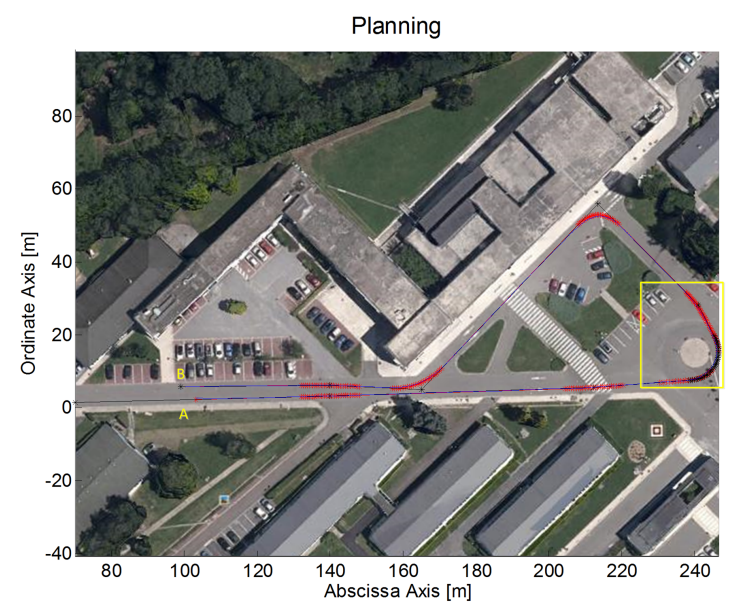

(a) Inria's facilities

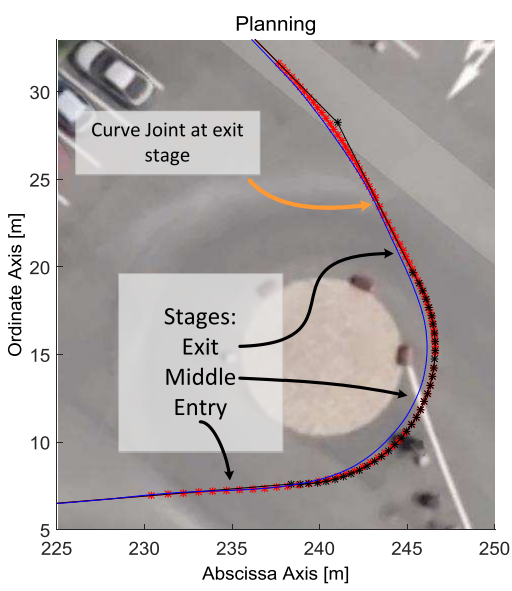

(b) Inria's facilities (zoom)

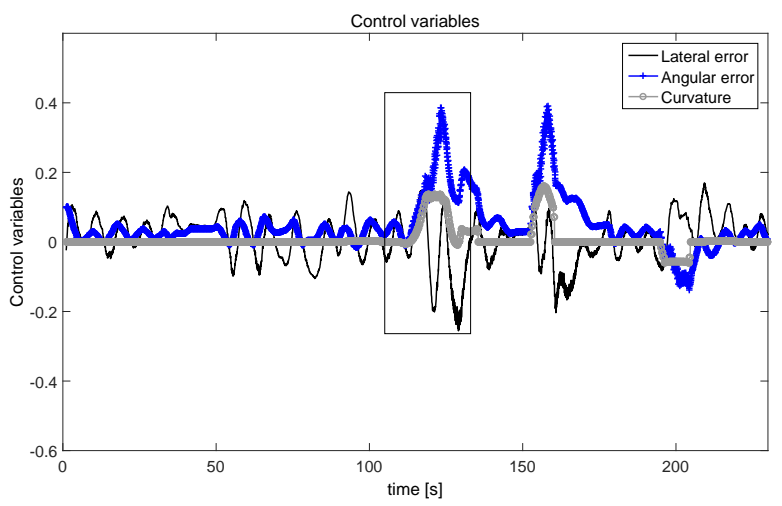

(c) Lateral control variables

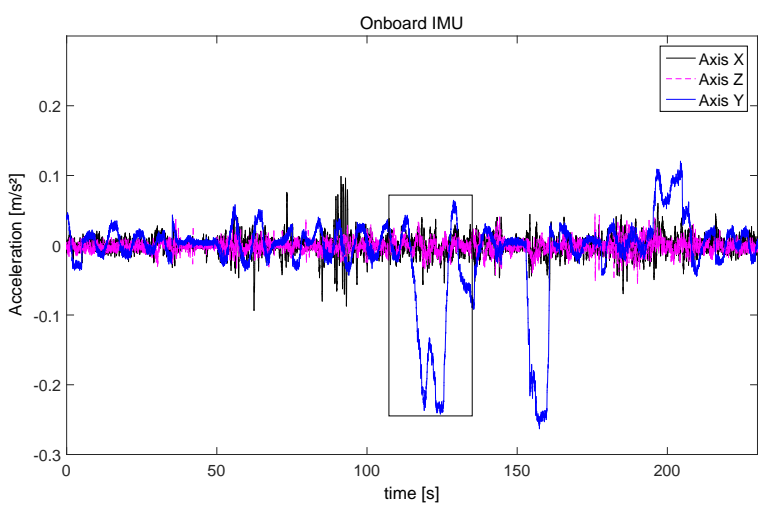

(e) On-board IMU

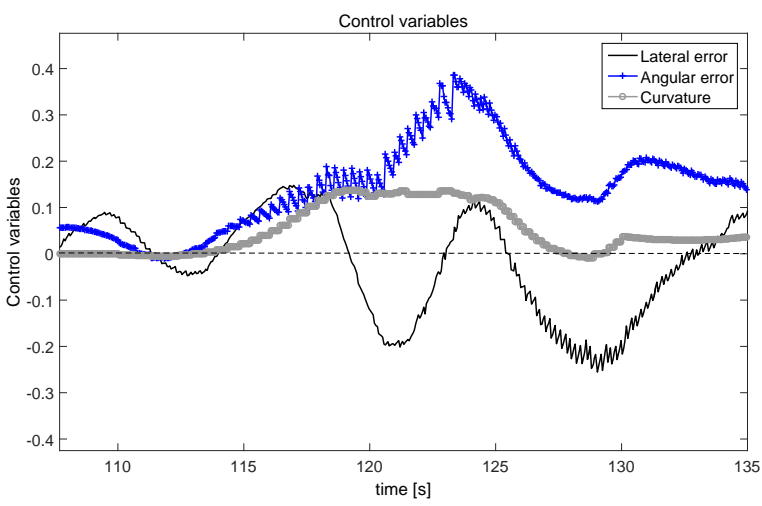

(d) Lateral control variables (zoom)

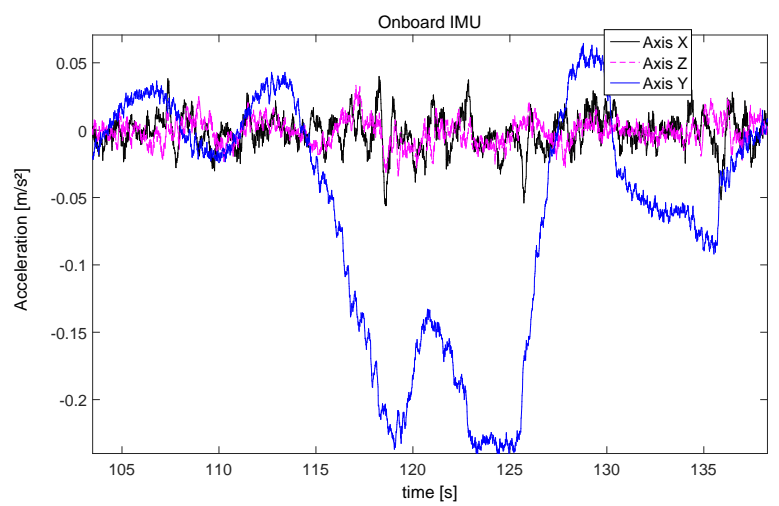

(f) On-board IMU (zoom)

Figure 7: Real urban scenario: Roundabout and intersections. 


\subsection{Real tests}

The proposed path planning for roundabouts was implemented on a Cyberbus and tested at Inria's facilities (see Figure 7(a)). The chosen route contains straight segments, urban intersections and a roundabout. This experiment was carried at $8 \mathrm{~km} / \mathrm{h}$, which is a normal velocity for these kind of platforms (Cybernetic Transportation Systems - CTS please see (González and Pérez, 2013)). It shows how the vehicle (black line) follows the reference generated by the path planning (in red).

Figure 7(c) shows the behavior of the control variables, displaying the lateral and angular error and the curvature. The lateral error is low and it keeps within a low value of $25 \mathrm{~cm}$, see Figure 7(d) (keep in mind that the implemented SLAM system has an error of about $15 \mathrm{~cm}$ ). Figure 7(e) shows the evolution of the accelerations read from the onboard IMU. The continuous line shows the lateral acceleration, which increase when the vehicle enters the roundabout (see Figure 7(f)), but always keeping a comfortable driving (accelerations under $0.35 \mathrm{~m} / \mathrm{s}^{2}$ (ISO, 1997)).

Figure 7(b) shows the real-time roundabout path generation and depicts the three stages: entrance, circulatory roadway and exit. The entrance stage differs from simulations in that the entry angle is tangent rather than perpendicular to the circulatory roadway. This angle information is taken from the digital global map. In Figure 7(d) the curvature (in grey) grows up to $1 / r$ (from 113 to 118 seconds approximately), where $r$ is the radius of the roundabout ( 8.5 meters for the present case). The circulatory roadway keeps the curvature constant. The exit stage shows how the generation of the curve can handle curved exit paths. The curvature of the exit stage is depicted between seconds 123 to 130 of Figure 7(d). Please notice that it reaches the curvature value of the next curved segment and a G1 behavior depicted at the curve joint in Figure 7(b). The curve generation time for each intersection is around $2 \mathrm{~ms}$ (milliseconds) for more than 2000 curves evaluated in a core duo Intel system $(2.0 \mathrm{GHz})$.

\section{CONCLUSIONS}

This paper presents a new path-planning algorithm for roundabouts. It generates a real-time path considering the specific roundabout constraints and the vehicle dynamics at low speeds. The proposed method is based on parametric equations, showing good results in a real implementation on a Cyberbus.

The proposed real-time path planning relies on road and vehicle constraints. Specifically, it considers the radius, central point of the roundabout and the exit to be taken on the roundabout. The path-planning generation was split in three stages: entrance, circulatory roadway and exit. Each part has a specific path generation based on parametric curves. Emergency situation can be handled through obstacle avoidance modules and a real time recalculation of the path (see (Gonzalez et al., 2014) for details).

Different exits of the roundabout were considered based on the French Road Circulation Code showing a comfortable performance, validating the proposed algorithm. Future work will be focused on the interaction with other vehicles on the roundabout.

\section{Acknowledgments}

Authors wants to express their gratitude to the FP7 EU AutoNet2030 project for its support in the development of this work.

\section{References}

Abaza, O. A., Hussein, Z. S., September 2009. Comparative analysis of multilane roundabout capacity "case study". In: IEEE 70th Vehicular Technology Conference Fall (VTC 2009-Fall). pp. 1-5.

Bai, Y., Xue, K., Yang, X., May 2009. Block mechanism of left-turned flow at signal-controlled roundabout. In: WRI Global Congress on Intelligent Systems. Vol. 3. pp. 443-449.

Bouraoui, L., Boussard, C., Charlot, F., Holguin, C., Nashashibi, F., Parent, M., Resende, P., 2011. An on-demand personal automated transport system: The citymobil demonstration in la rochelle. IEEE Intelligent Vehicles Symposium 4, $1086-1091$.

Brezak, M., Petrovic, I., April 2014. Real-time approximation of clothoids with bounded error for path planning applications. IEEE Transactions on Robotics 30 (2), 507-515.

Broggi, A., Buzzoni, M., Debattisti, S., Grisleri, P., Laghi, M., Medici, P., Versari, P., Sept 2013. Extensive tests of autonomous driving technologies. IEEE Transactions on Intelligent Transportation Systems 14 (3), 1403-1415.

Broggi, A., Cerri, P., Debattisti, S., Laghi, M. C., Medici, P., Panciroli, M., Prioletti, A., 2014. Proud-public road urban driverless test: Architecture and results. In: 2014 IEEE Intelligent Vehicles Symposium Proceedings. IEEE, pp. 648-654. 
De Brabander, B., Vereeck, L., 2007. Safety effects of roundabouts in flanders: Signal type, speed limits and vulnerable road users. Accident Analysis and Prevention.

Ferguson, D., Howard, T., Likhachev, M., 2009. Motion planning in urban environments. In: Buehler, M., Iagnemma, K., Singh, S. (Eds.), The DARPA Urban Challenge. Vol. 56 of Springer Tracts in Advanced Robotics. Springer Berlin Heidelberg, pp. 61-89.

Godoy, J., Perez Rastelli, J., Onieva, E., Villagra, J., Milanés, V., Haber, R., 2015. A driverless vehicle demonstration on motorways and in urban environments. Transport.

González, D., Pérez, J., 2013. Control architecture for cybernetic transportation systems in urban environments. In: IEEE Intelligent Vehicles Symposium. pp. 1119-1124.

Gonzalez, D., Perez, J., Lattarulo, R., Milanes, V., Nashashibi, F., Oct 2014. Continuous curvature planning with obstacle avoidance capabilities in urban scenarios. In: IEEE 17th International Conference on Intelligent Transportation Systems (ITSC). pp. 1430-1435.

González, D., Pérez, J., Milanés, V., Nashashibi, F., 2016. A review of motion planning techniques for automated vehicles. IEEE Transactions on Intelligent Transportation Systems 17 (4), 1135-1145.

Hand, L., Yashiro, H., Nejad, H., Do, Q. H., Mita, S., June 2010. Bézier curve based path planning for autonomous vehicle in urban environment. IEEE Intelligent Vehicles Symposium 4, 1036 - 1042.

Isebrands, H., 2009. Roundabouts and signals: Harmony even with increasing traffic volumes current guidance. ITE Journal.

ISO, 1997. Mechanical vibration and shock-evaluation of human exposure to whole-body vibration-part 1: General requirements. Tech. Rep. ISO 2631-1, International Organization for Standardization.

Johnson, M., Hange, W., 2003. Modern roundabout intersections: When to use them? a comparison with signalized intersections. Tech. rep., Institute of Transportation Engineers.

Labakhua, L., Nunes, U., Rodrigues, R., Leite, F. S., 2008. Smooth trajectory planning for fully automated passengers vehicles: spline and clothoid based methods and its simulation. In: Informatics in Control Automation and Robotics. Springer, pp. 169-182.

Manage, S., Nakamura, H., Suzuki, K., 2003. Performance an of roundabouts as an alternative for intersection control in japan. Journal of the Eastern Asia Society for Transportation Studies 5, 871-883.

Milanés, V., Villagra, J., Godoy, J., Simó, J., Pérez, J., Onieva, E., 2012. An intelligent v2i-based traffic management system. IEEE Transactions on Intelligent Transportation Systems 13 (1), 49-58.

Molinete, B., Bouraui, L., Naranjo, J., Kostense, H., Hendriks, J., Alonso, J., Lobrino, R., Isasi, L., 2009. Cybercars-2: Close communications for cooperation between cybercars. Tech. rep., Technical Report Project No IST-2004-0228062.

Muffert, M., Milbich, T., Pfeiffer, D., Franke, U., June 2012. May i enter the roundabout? a time-to-contact computation based on stereo-vision. In: Intelligent Vehicles Symposium (IV), 2012 IEEE. pp. 565-570.

Naranjo, J. E., Bouraoui, L., García, R., Parent, M., , Sotelo, M. A., March 2009. Interoperable control architecture for cybercars and dual-mode cars. In: IEEE Transactions on Intelligent Transportation Systems. Vol. 10. pp. $146-154$.

Paden, B., Cap, M., Yong, S. Z., Yershov, D., Frazzoli, E., 2016. A survey of motion planning and control techniques for self-driving urban vehicles. arXiv preprint arXiv:1604.07446.

Pérez, J., Milanés, V., de Pedro, T., Vlacic, L., 2011. Autonomous driving manoeuvres in urban road traffic environment: a study on roundabouts. In: Proceedings of the 18th World Congress The International Federation of Automatic Control. pp. 13795-13800.

Perez Rastelli, J., Lattarulo, R., Nashashibi, F., June 2014. Dynamic trajectory generation using continuous-curvature algorithms for door to door assistance vehicles. In: IEEE Intelligent Vehicles Symposium Proceedings. pp. 510-515.

Piazzi, A., Lo Bianco, C. G., Bertozzi, M., Fascioli, A., Broggi, A., August 2002. Quintic g2-splines for the iterative steering of vision-based autonomous vehicles. IEEE Transactions on Intelligent Transportation Systems 3, 27 - 36.

Rice, E., 2010a. Mini-roundabouts. Tech. rep., U.S. Departmet of Trasportation Federal Highway Administration (FHWA-SA-10-007)

Rice, E., 2010b. Roundabouts. Tech. rep., U.S. Departmet of Trasportation Federal Highway Administration (FHWA-SA-10-006).

Robinson, B. W., Rodegerdts, L., Scarbrough, W., Kittelson, W., Troutbeck, R., Brilon, W., Bondzio, L., Courage, K., Kyte, M., Mason, J., Flannery, A., Myers, E., Bunker, J., G., J., 2000. Roundabouts: An informational guide. Tech. rep., Report FHWA-RD-00-067. FHWA, U.S. Department of Transportation.

Rodegerdts, L., Bansen, J., Tiesler, C., Knudsen, J., Myers, E., 2010. Roundabouts: An informational guide. Tech. rep., National Cooperative Highway Research Program.

Roldao, L., Perez Rastelli, J., González, D., Milanés, V., 2015. Description and technical specification of cybernetic transportation systems: an urban transportation concept -citymobil2 project approach-. In: IEEE ITSC. pp. 176-181.

Sotelo, M. A., 2003. Lateral control strategy for autonomous steering of ackerman-like vehicles. Robotics and Autonomous Systems 45,223 - 233.

Tracza, M., Chodura, J., 2012. Performance and safety of roundabouts with traffic signals. In: SIIV 5th International Congress Sustainability of Road Infrastructures. pp. $789-800$.

Trehard, G., Alsayed, Z., Pollard, E., Bradai, B., Nashashibi, F., Sept 2014. Credibilist simultaneous localization and mapping with a lidar. In: IEEE/RSJ International Conference onIntelligent Robots and Systems (IROS 2014). pp. 2699-2706.

Van Schijndel-de Nooij, W., Krosse, B., Van den, T., Maas, S., Van Nunen, E., Zwijnenberg, H., Schieben, A., Mosebach, H., Ford, N., McDonald, M., Jeffery, D., Piao, J., Sanchez, J., 2011. Definition of necessary vehicle and infrastructure systems for automated driving. Tech. rep., Study Report European Commission.

Xiaoguang, Y., Xiugang, L., Kun, X., 2004. A new traffic-signal control for modern roundabouts method and application. IEEE Intelligent Transportation Systems Society 5, $282-287$.

Xie, J., Nashashibi, F., Parent, M., Favrot, O. G., December 2010. A real-time robust global localization for autonomous mobile robots in large environments. In: 11th International Conference on Control Automation Robotics \& Vision (ICARCV). Singapore, pp. $1397-1402$.

Yu, L., Qin, C., Sept 2009. Real-time signal control method for multi-approach roundabouts. In: International Conference on Management and Service Science (MASS). pp. 1-4. 
Ziegler, J., Bender, P., Schreiber, M., Lategahn, H., Strauss, T., Stiller, C., Dang, T., Franke, U., Appenrodt, N., Keller, C., Kaus, E., Herrtwich, R., Rabe, C., Pfeiffer, D., Lindner, F., Stein, F., Erbs, F., Enzweiler, M., Knoppel, C., Hipp, J., Haueis, M., Trepte, M., Brenk, C., Tamke, A., Ghanaat, M., Braun, M., Joos, A., Fritz, H., Mock, H., Hein, M., Zeeb, E., Summer 2014. Making bertha drive-an autonomous journey on a historic route. IEEE Intelligent Transportation Systems Magazine 6 (2), 8-20. 